\title{
Elitenrekrutierung und die Qualität politischer Führung
}

Elitenrekrutierung ist eine der zentralen Aufgaben politischer Parteien. Moderne Demokratien sind repräsentative Demokratien, d. h. Bürger übergeben die Entscheidungen über politische Sachverhalte auf Zeit einer relativ kleinen Zahl gewählter Repräsentanten. Diese Gewählten - häufig werden sie auch als ein Teil der politischen Klasse oder politischen Elite ${ }^{2}$ bezeichnet - übernehmen im politischen System auf Zeit bestimmte Aufgaben der Leitung, Koordination und Planung, kurzum: sie übernehmen bestimmte Funktionen. Otto Stammer ${ }^{3}$ hat bereits in den fünfziger Jahren den Begriff der Funktionselite geprägt und folgendermaßen definiert: »Eliten sind die mehr oder weniger geschlossenen sozialen und politischen Einflussgruppen, welche sich aus den breiten Schichten der Gesellschaft und ihren größeren und kleineren Gruppen auf dem Wege der Delegation oder der Konkurrenz herauslösen, um in der sozialen oder der politischen Organisation des Systems eine bestimmte Funktion zu übernehmen. « ${ }^{4}$ Obwohl Parteien die zentrale Rekrutierungsstätte für die politische Elite darstellen und zahlreiche Parteienforscher diese Funktion sogar in ihre Definition politischer Parteien integrieren ${ }^{5}$, hat sich die Parteienforschung mit theoretischen Konzeptionen der innerparteilichen Rekrutierung wenig beschäftigt. Es ist die Elitenforschung, die Antworten auf die Fragen nach der Rekrutierung (Woher kommen die Eliten?) und der Integration (Wie repräsentieren sie?) geben. In den wenigen Arbeiten der Parteienforschung stehen Fragen nach den Auswahlmechanismen (Wie wird man Kandidat?) im Mittelpunkt. Dabei wird zu-

1 Dieser Aufsatz ist die überarbeitete Fassung meines Habilitationsvortrages, gehalten am 17. Januar 2001 an der FU Berlin. Für konstruktive Kritik und wichtige Hinweise möchte ich Hans-Dieter Klingemann, Bernhard Weßels, Daniele Caramani und Lars Leiner danken.

2 Zur Differenzierung zwischen politischer Klasse und politischer Elite vgl. Klaus von Beyme, Die politische Klasse im Parteienstaat, Frankfurt a. M. 1993, S. 11-24.

3 Otto Stammer, "Das Elitenproblem in der Demokratie in: Schmollers Jabrbuch für Gesetzgebung, Verwaltung und Volkswirtschaft, Jg. 71/1951, S. 513-540, hier S. 514.

4 Eine funktionale Elitendefinition erscheint nicht nur für empirische Untersuchungen brauchbarer als der Begriff der Machtelite, denn eine solche Definition enthält ein Kriterium, an dem sich messen lässt, inwieweit bestimmte Verfahren der Elitebildung und Führerauslese zum Erfolg führen: nämlich die optimale Erfüllung einer bestimmten Funktion. Der Begriff der Machtelite hingegen beinhaltet lediglich die Annahme, dass Macht in Gesellschaften ungleich verteilt ist, er sagt jedoch nichts über die Gestalt und die Merkmale einer gesellschaftlichen Elite aus.

5 V. O. Key, American State Politics: An Introduction, New York 1956 ; Giovanni Sartori, Parties and Party Systems. A Framework for Analysis, Cambridge 1976; Joseph Schlesinger, Political Parties and the Winning of Office, Ann Arbor 1991. 
nehmend festgehalten, dass die Personalrekrutierung innerhalb der Parteien - auch nach Aussage der eigenen Führungskräfte - sehr planlos verläuft und im allgemeinen eine neue, sachgerechte Entscheidung für die Personalrekrutierung gefordert wird.

Die vorliegende Studie möchte einen Beitrag zur Schließung der mangelnden theoretischen Fundierung von Auswahlmechanismen und deren Zusammenhang mit Qualitätscharakteristiken der rekrutierten Eliten leisten. Die zentrale Fragestellung lautet: Welche idealtypischen Nominierungsverfahren, d. h. unterschiedliche Mechanismen zur Rekrutierung und Selektion von Kandidaten für politische Ämter gibt es und welche unterschiedlichen Führungsqualitäten bringen diese hervor. Die Studie lässt sich auf der Schnittstelle dreier Forschungsstränge in der politischen Soziologie verorten, der Parteien-, der Eliten- sowie der Wahlforschung. Ferner ist sie geprägt von einer institutionalistischen Perspektive, die davon ausgeht, dass Einstellungen, Eigenschaften und das Verhalten der politischen Elite in ihrem jeweiligen institutionellen Kontext verstanden werden muss $^{6}$. "The new institutionalism approach ... assumes that the politics of recruitment is not simply reducible to either the attitudes, preferences and concerns of individual actors, or the legal and constitutional structures within which they work. Instead, there is a process of interaction: the rules and procedures of political systems structure behaviour, attitudes and opinions in predictable and orderly ways. «7

Die Studie gliedert sich in vier Teile: Während in einem ersten Teil die in der Eliten- und Parteienforschung verwandten Termini der Rekrutierung und Selektion begrifflich klar definiert und voneinander abgegrenzt werden, werden in einem darauffolgenden Schritt unterschiedliche Mechanismen zur Rekrutierung und Selektion politischer Kandidaten theoretisch diskutiert und in einer Typologie zusammengeführt. Die sich daraus ergebenden Führungseigenschaften politischer Kandidaten werden in einem dritten Teil des Beitrags vor dem Hintergrund der Ergebnisse der jüngeren Studien zur Wahlforschung, die sich u. a. auch mit den Kandidatenmerkmalen und -eigenschaften beschäftigen, erörtert. Abschließend werden die unterschiedlichen Führungsqualitäten zum Funktionieren repräsentativer Demokratien diskutiert und die Auswahlmechanismen beispielhaft verschiedenen Ämtern zugewiesen.

\section{Selektion und Rekrutierung - Eine Begriffsklärung}

Der Begriff der Elitenrekrutierung wird in der Eliten- und Parteienforschung für teilweise unterschiedliche Sachverhalte benutzt. In zahlreichen Elitestudien wird

6 James March / Johan Olson, Rediscovering Institutions, New York 1989; Paul Di Maggio / Walter W. Powell, The New Institutionalism in Organizational Analysis, Chicago 1991.

7 Pippa Norris, »Introduction: Theories of Recruitment « in: Pippa Norris (Hg.), Passages to Power. Legislative Recruitment in Advanced Democracies, Cambridge 1997, S. 1-14, hier S. 9. 
unter Rekrutierung die Identifizierung sozialer und ökonomischer Charakteristika der untersuchten Eliten verstanden. »Wer gehört in Deutschland zur Elite? Aus welchen Elternhäusern kommen die Führungsgruppen in Wirtschaft und Politik, Wissenschaft, Verwaltung, Kultur, den Gewerkschaften, den Verbänden und den Massenmedien? Welche Ausbildungen und welche Karrierewege führen in die rund 4000 höchsten Entscheidungspositionen in Deutschland? Ist die nationale Führungsschicht eine geschlossene Gesellschaft, die nur Personen mit elitärer Herkunft und entsprechenden Wertorientierungen kooptiert? Oder ist die Positionselite offen für Aufsteiger auch aus unteren Sozialschichten oder Personen mit neuen, unkonventionellen Einstellungen zu Politik und Gesellschaft? ... Wie gut integriert die deutsche Führungsschicht die unterschiedlichen sozialen Gruppen und Wertgemeinschaften in Deutschland? « So formuliert die Potsdamer Elitestudie unter der Leitung von Wilhelm Bürklin die zentralen Fragen der Eliteforschung. Generell lassen sich hier unterschiedliche Forschungsrichtungen identifizieren?: Die ersten Elitestudien stellten die Frage nach der Herkunft der Eliten bzw. nach dem Ausmaß des Austausches nationalsozialistischer Eliten ${ }^{10}$. Gefragt wurde hier nach sozialen Charakteristika und Karrieremustern bzw. -verläufen. Die Offenheit der Elitenrekrutierung ist vielfach als ein wichtiges Merkmal demokratischer Eliten betrachtet worden; nachdem zwar die Zugangschancen formal gesichert wurden, ist zu prüfen, inwieweit diese auch faktisch gegeben sind. Diese social background analysis wurde auch in die Abgeordnetenforschung übernommen. In regelmäßigen Abständen werden meist in Handbuchformat Daten zur Sozialstruktur der Parlamente vorgelegt $\mathrm{t}^{11}$. Auch die Frage der Repräsentation von Frauen in Parlamenten und hiermit verbunden die Diskussion, ob Repräsentation als »sozialstrukturelle Verankerung von Interessen « verstanden werden kann, oder ob es sich nicht eher um »die politische Vermittlung von Interessen handelt $\ll^{12}$, lässt sich diesem Forschungszweig zuordnen.

Einen anderen Zungenschlag finden wir in der Parteienforschung. Hier spielt nicht die Frage »Woher kommen die Kandidaten«, sondern »Wie kommen die Kan-

8 Wilhelm Bürklin / Hilke Rebenstorf u. a., Eliten in Deutschland. Rekrutierung und Integration, Opladen 1997; Ursula Hoffmann-Lange, Eliten, Macht und Konflikt in der Bundesrepublik Deutschland, Opladen 1992; Rudolf Wildenmann, »Unsere Oberen Dreitausend. Woher kommen sie? Was prägt sie? Was wollen sie? Eine Eliten-Studie« in: Die Zeit Nr. 10/1982.

9 Vgl. auch Bürklin/Rebenstorf, aaO. (FN 8), S. 39; Hoffmann-Lange, aaO. (FN 8), S. 19.

10 Lewis J. Edinger, »Post-Totalitarian Leadership: Elites in the German Federal Republic « in: American Political Science Review Vol. 54 (1960), S. 58-82; Lewis J. Edinger, »Continuity and Change in the Background of German Decision-Makers « in: The Western Political Quarterly Vol. 14 (1961), S. 17-36; Wolfgang Zapf, Wandlungen der Deutschen Elite, München 1965.

11 Für die BRD Peter Schindler, Datenhandbuch zur Geschichte des Deutschen Bundestages, Baden-Baden 1984, 1986, 1988, 1994.

12 Suzanne Schüttemeyer, »Parlamentarische Interessensvermittlung: Lücken in der Repräsentation und Veränderungen im Verfassungskonsens « in: Ralf Kleinfeld / Wolfgang Luthardt (Hg.), Westliche Demokratien und Interessensvermittlung, Marburg 1993, S. 47-58, hier S. 50. 
didaten an ihre Position « eine zentrale Rolle. Es stehen also die Auswahlmechanismen im Mittelpunkt. Während Max Weber noch das Parlament als die zentrale Ausbildungsstätte für Politiker betrachtete, stellen heute nahezu ausnahmslos politische Parteien die wichtigste »Lehrstätte « für (angehende) Politiker dar. Parteien stellen in modernen hochgradig differenzierten Demokratien die politischen Rekrutierungskanäle dar. Der Grund hierfür liegt in der zunehmenden Differenzierung moderner Gesellschaften und der damit einhergehenden Professionalisierung. Politiker müssen über besondere Qualitäten verfügen, um im politischen Geschäft bestehen zu können. Politik ist zur Profession geworden. Die interne Organisation politischer Parteien in Verbindung mit ihrer externen Verkoppelung mit Verbänden und Institutionen bestimmt den Grad an »Offenheit des politischen Führungsauswahlprozesses in großem Maße. Deshalb ist es erforderlich, politische Führungsauswahl im Kontext der Parteiorganisation zu analysieren. $\ll^{13}$

Herzog ${ }^{14}$ unterscheidet in dem von ihm entwickelten Modell vier Phasen im Verlauf der politischen Elitebildung: (1) die Sozialisationsphase, (2) die Phase der politischen Rekrutierung, zumeist durch Parteibeitritt, (3) die Phase der politischen Karriere und (4) die Phase der Elitenrekrutierung im engeren Sinne, d. h. die Rekrutierung von Personen aus » Vorpositionen « in die (jeweils untersuchten) Spitzenpositionen. Auch verwendet er in seinen Analysen einen breiter gefassten Rekrutierungsbegriff. Er spricht von den »Mechanismen der Führungsrekrutierung «, die politisches Personal hervorbringt ${ }^{15}$. Diese Perspektive beleuchtet neben den sozialen und ökonomischen Eigenschaften der politischen Elite auch die Auswahlmechanismen.

Die Parteienforschung konzentriert sich vor allem auf die vierte Phase des Modells, d. h. auf die Rekrutierung von Vorpositionen auf Spitzenpositionen. Aber auch hier werden die Termini Rekrutierung und Selektion häufig synonym verwendet. Norris ${ }^{16}$ spricht in ihrem Band mit dem treffenden Titel »Passages to Power « von einem »recruitment process ... as a structured market-place. We distinguish between components of opportunities set by the political system, the rule-governed process of recruitment within parties, the demands of each party, and the supply of candidates. ${ }^{17}$ Die Zeitschrift Party Politics widmet der Rekrutierung und Selektion von politischen Kandidaten ein Sonderheft mit dem Titel »Democratizing Candidate Selection: Causes and Consequences «, wobei die Herausgeber unter Selektion unterschiedliche Auswahlmechanismen verstehen ${ }^{18}$. Auch der Sammelband von Marsh und Gallagher ${ }^{19}$ bedient sich des Begriffes der Kandidatenselektion und widmet sich in einzelnen Länderstudien der Prozedur, mit welcher politische Parteien

13 Dietrich Herzog, Politische Karrieren. Selektion und Professionalisierung politischer Führungsgruppen, Opladen 1975, S. 13.

14 Ebd., S. 48.

15 Dietrich Herzog, »Der moderne Berufspolitiker. Karrierebedingungen und Funktion in westlichen Demokratien« in: Der Bürger im Staat, Band 1991/1992, S. 28-51, hier S. 28.

16 Norris, aaO. (FN 7).

17 Ebd., S. 11.

18 Party Politics, Vol. 7, 3/2001, S. 3. 
ihre Kandidaten für nationale Parlamente aufstellen. Die aufgeführten Arbeiten weisen alle auf den mehrstufigen Prozess der Elitenrekrutierung hin. Diesem Sachverhalt trägt der vorliegende Beitrag Rechnung und unterscheidet im Prozess der Elitenrekrutierung theoretisch zwischen Rekrutierung und Selektion als zwei aufeinanderfolgende Vorgänge. Unter Rekrutierung wird die Bildung eines Pools verstanden, aus dem die Kandidaten geschöpft werden. Unter Selektion wird der Auswahlmechanismus sowie das Auswahlgremium verstanden - wer wählt die Kandidaten wie aus?

\section{Rekrutierung}

Wenden wir uns der Rekrutierung zu. Wir fragen also zunächst, aus welchem »Pool« das politische Führungspersonal stammt. Hier herrscht Einigkeit darüber, dass es in modernen Demokratien in erster Linie politische Parteien sind, die den Rekrutierungsprozess kanalisieren. Betrachtet man die vier zentralen Funktionen politischer Parteien, nämlich die Zielfindungsfunktion, die Artikulation und Aggregation gesellschaftlicher Interessen, die Mobilisierung und Sozialisation der Bürgerinnen und Bürger und die Elitenrekrutierung, so kommt letzterer in repräsentativen Demokratien im historischen Vergleich eine immer wichtigere und bedeutendere Rolle zu. »The nominating process ... has become the crucial process of the party. The nature of the nominating procedure determines the nature of the party; he who can make the nominations is the owner of the party. This is therefore one of the best points at which to observe the distribution of power within the party. $\ll^{20}$ Die zunehmende gesellschaftliche Differenzierung und Komplexität politischer Sachverhalte fordert von den politischen Akteuren ein immer größeres Maß an Fachwissen, an fachlicher Spezialisierung sowie den gelernten Umgang mit den institutionellen Komplexitäten von Politikprozessen. Politik wird bzw. muss als Beruf verstanden werden und Parteien sind der Ort, sich diese beruflichen Qualifikationen anzueignen. So steigt auch der Anteil der Politiker immer weiter, der seine politische Laufbahn bereits frühzeitig, oft schon in der Ausbildungsphase beginnt und unmittelbar in eine besoldete politische Berufstätigkeit einsteigt. Dieser Politikertypus ist von Anbeginn ein Professional21, materiell gebunden und psycholo-

19 Michael Marsh / Michael Gallagher (Hg.), Candidate Selection in Comparative Perspective: The Secret Garden of Politics, London 1988.

20 Elmer E. Schattschneider, Party Government, New York 1942, S. 64; siehe auch Otto Kirchheimer, »The Transformation of the Western European Party Systems « in: Joseph Lapalombara / Myron Weiner (Hg.), Political Parties and Political Development, Princeton 1966, S. 177-200, hier S. 198 f.; Klaus von Beyme, Der Gesetzgeber: Der Bundestag als Entscheidungszentrum, Opladen 1997, S. 381; Richard Katz / Peter Mair, »Changing Models of Party Organization and Party Democracy: The Emergence of the Cartel Party« in: Party Politics, Vol. 1, 1/1995, S. 5-28; Richard Katz, »The Problem of Candidate Selection and Models of Party Democracy « in: Party Politics, Vol. 7, 3/2001, S. 277-296.

21 Im Gegensatz zur Standard-Karriere oder zur Cross-Over-Karriere, vgl. hierzu Herzog, aaO. (FN 13), S. 41. 
gisch adaptiert an den politischen Beruf als seine einzige, gewöhnlich lebenslange Einnahmequelle. Natürlich lassen sich vor allem auf lokaler Ebene auch parteiunabhängige Kandidaturen beobachten, alle Ebenen des politischen Prozesses betrachtend stellt diese Form der Rekrutierung jedoch die Ausnahme dar. So vermerkte auch Richard von Weizsäcker (2003) jüngst in der ZEIT, dass sich Parteien mittlerweile zu machtvollen Maschinerien der Rekrutierung entwickelt haben. "Systematisch ausgebaut und sorgfältig abgeschirmt haben sie ihr Monopol beim Zugang zu politischen Mandaten und Ämtern. ... Es sind die Parteien und ihre Fraktionen, die exklusiv über einen politischen Aufstieg oder Ausschluss entscheiden."

In der Literatur werden auch Variationen dieses Prozesses angesprochen. So weist Gallagher ${ }^{22}$ auf den Zentralisierungsgrad der Kandidatenrekrutierung hin: "How centralized is the process? « Rahat/Hazan ${ }^{23}$ sprechen im Hinblick auf Unterschiede bei der Kandidatenrekrutierung (wobei sie den Begriff der candidate selection wählen) von einem »level of inclusiveness or exclusiveness«. Für unsere Zwecke wollen wir Variationen im Rekrutierungsprozess folgendermaßen systematisieren:

- Der Rekrutierungsprozess kann offener gestaltet sein, d. h. es ist leichter und zeitunaufwendiger für einen Kandidaten, innerhalb der Parteiorganisation in das Pool der Bewerber zu gelangen. Er hat weniger Ämter, Positionen und Funktionen zu durchlaufen. Die Parteistruktur weist insgesamt eine höhere Permeabilität auf. Im Extremfall muss der Kandidat nicht einmal Mitglied einer Partei sein, um in das Pool der Bewerber zu gelangen.

- Bei einer eher geschlossenen Rekrutierung ist dagegen zu erwarten, dass der Kandidat schon eine ganze Reihe von Funktionen und Posten innerhalb der Parteiorganisation durchschritten haben muss, um zur Nominierung zu gelangen. Eine längere innerparteiliche Karriere ist Bedingung für die politische Qualifikation. Die Parteistruktur weist also insgesamt eine geringere Permeabilität auf. So beobachtet Obler ${ }^{24}$ in den frühen siebziger Jahren bei den belgischen Sozialisten eine relativ geschlossene Form der Selektion mit klar formulierten Bedingungen an den potenziellen Kandidaten. Er muss »(1) have been a member of at least five years prior to the primary, (2) have made annual minimum purchases from the socialist co-op; (3) have been a regular subscriber to the party's newspaper; (4) have sent his children to state rather than Catholic schools; and (5) have his wife and children enrolled in the appropriate women's and youth organizations. "Graphik 1 verdeutlicht diesen Sachverhalt.

22 Michael Gallagher, »Introduction « in: Michael Marsh / Michael Gallagher (Hg.), Candidate Selection in Comparative Perspective: The Secret Garden of Politics, London 1988, S. 1-19, hier S. 1.

23 Gideon Rahat / Reuven Y. Hazan, »Candidate Selection Models: An Analytical Framework « in: Party Politics, Vol. 7, 3/2001, S. 297-322, hier S. 300.

24 Jeffrey Obler, »Intraparty Democracy and the Selection of Parliamentary Candidates: The Belgian Case in: British Journal of Political Science 4 (1974), S. 163-185, hier S. 180. 


\section{Graphik 1: Rekrutierung politischer Kandidaten}

\begin{tabular}{|c|c|c|c|}
\hline Alle Bürger & Parteimitglieder & $\begin{array}{l}\text { Parteimitglieder } \\
\text { mit bestimmten } \\
\text { Erfahrungen }\end{array}$ & $\begin{array}{l}\text { Pateimitglieder, } \\
\text { die zahlreiche } \\
\text { Ämter und } \\
\text { Positionen durch- } \\
\text { laufen müssen }\end{array}$ \\
\hline
\end{tabular}

Offen

geschlossen

Wenden wir uns nun dem Aspekt der Selektion zu, d. h. der Frage: Wer wählt wie aus?

\section{Selektion}

An der Selektion der Kandidaten wird häufig gemessen, wie offen und demokratisch der gesamte Prozess der Kandidatenaufstellung abläuft und inwiefern innerparteiliche Demokratie gesichert ist. »The way in which political parties select their candidates may be used as an acid test of how democratically they conduct their internal affairs. ${ }^{25}$ Als den demokratietheoretischen Standards am ehesten entsprechend erscheint die Selektion der Kandidaten unmittelbar durch die Wähler. Vor allem Demokratietheorien mit partizipatorischer Schlagseite, die auf die Maximierung von Partizipationsmöglichkeiten setzen, fordern eine solche Form der Selektion. Bei den Parteien selbst finden solche Forderungen weniger Rückhalt, da sie mit einem Funktionsverlust politischer Parteien, quasi einer Aushöhlung, einhergehen. Eine der zentralen Funktionen politischer Parteien ist die der Elitenrekrutierung und -selektion; hier stellt sich für die Partei(-spitze) lediglich die Frage, wer innerhalb der Partei den Kandidaten (aus-)wählt. Eine Möglichkeit besteht darin, den Kandidaten direkt über alle Parteimitglieder, d. h. über die membership party, zu wählen. Diese Vorgehensweise würde sich auf der Selektionsdimension zwischen offener und geschlossener Selektion einordnen lassen (vgl. Graphik 2). Unter einer eher geschlossenen Selektion ist die Ernennung eines Kandidaten von Seiten der Parteispitze bzw. -führung zu verstehen. Arbeiten, die den Wettbewerbscharakter und die häufig damit verbundenen Zentralisierungstendenzen politischer Parteien betonen, diskutieren die Auswahl der Kandidaten seitens der Parteispitze, also eine Selektion »von oben«. Hierzu zählen sowohl Demokratietheorien mit elitistischer Einfärbung als auch jüngere Arbeiten aus der Parteienforschung ${ }^{26}$. Aus einer wettbewerbstheoretischen Perspektive wird das sich verändernde Verhältnis zwischen Parteien und Wählern und die daraus resultierende veränderte Logik der Selektion unterstrichen: Aufgrund eines Wandels des Wählerverhaltens, der Lockerung bzw. Auflösung der bisher vorhandenen Par-

25 Gallagher, aaO. (FN 22), S. 1.

26 Angelo Panebianco, Political Parties: Organization and Power, Cambridge 1988; Katz / Mair, aaO. (FN 20). 
teibindungen rücken Wahlen immer mehr in den Vordergrund und somit auch die Frage nach politischem Führungspersonal, das sowohl die Parteimitglieder als auch einen Großteil der Wähler anspricht. Die Selektion des Kandidaten liegt also mit zunehmender Zentralisierung politischer Parteien de facto bei der Parteiführung, allerdings richtet sich die Logik der Selektion aus wettbewerbstaktischen Gründen mehr und mehr nach der (antizipierten) Einstellung der Wähler (vgl. Graphik 2).

\section{Graphik 2: Selektion politischer Kandidaten}

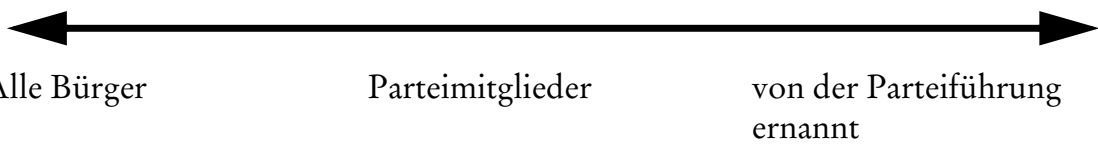

Offen

geschlossen

Es ist nicht ganz einfach, empirische Beispiele für die Rekrutierung und Selektion politischer Kandidaten anzuführen, da es häufig keine klaren, festgelegten Richtlinien und Regeln gibt, nach denen dieser Vorgang ablaufen soll. Zudem lassen sich die politischen Parteien hier ungern in die Karten schauen. In der Tat handelt es sich hier noch häufig um einen »secret garden of politics ${ }^{27}$. Als Beispiele eines offenen Selektionsprozesses können amerikanische primaries aufgeführt werden. Die Reformvorschläge der McGovern-Fraser Commission Anfang der siebziger Jahre nahm den Parteibossen den Einfluss auf die Nominierung der Präsidentschaftskandidaten. Zentraler Punkt der Reformen war die Absicht, die Entscheidung über die Präsidentschaftskandidaten der Parteien in die Hände der jeweiligen Partei-Wähler zu legen. Dies solle entweder über die bereits bekannten Vorwahlen oder über »Party Caucuses « erfolgen. Die Nominierung zum Präsidentschaftskandidaten setzt nun nicht mehr die Unterstützung durch die Parteielite voraus, sondern Siege in zahlreichen Vorwahlen, vor allem in jenen zu Beginn des innerparteilichen Wahlkampfes und in den einwohnerstarken Bundesstaaten. Nur so konnte es beispielsweise dem weitgehend unbekannten und nicht zum demokratischen Parteiestablishment zählenden Jimmy Carter gelingen, 1976 als Präsidentschaftskandidat seiner Partei nominiert zu werden. »Instead of making the traditional rounds among party leaders, Carter travelled about the country meeting with journalists ... Carter would not have won under the old rules. ${ }^{28} \mathrm{Zu}$ den Konsequenzen dieser Reform der Kandidatenselektion zählen neben der Aushöhlung der Partei und der Stärkung der einzelnen Kandidaten Veränderungen in der Wahlkampfführung. Wahlkämpfe werden nun unter Zuhilfenahme professioneller Beraterstäbe geführt, die dem Kandidaten und nicht der Partei unterstellt sind ${ }^{29}$. Diese Selektion bevorzugt Kandidaten aus

27 Marsh / Gallagher, aaO. (FN 19).

28 Thomas E. Patterson, Out of Order. How the Decline of the Political Parties and the Growing Power of the News Media Undermine the American Way of Electing Presidents, New York 1993, S. 32 f. 
den jeweiligen politischen Spitzenpositionen der Bundesstaaten im Vergleich zu Abgeordneten des Kongresses. »The wide-spread use of primaries in American presidential politics had shifted the balance toward candidates from state political arenas rather than Congress. $\ll^{30}$ Vier der letzten fünf amerikanischen Präsidenten hatten vor der Nominierung zum Präsidentschaftskandidaten ihrer Partei den Posten eines Gouverneurs inne und es war lediglich Ronald Reagan, der vor seiner Nominierung nationalen Bekanntheitsgrad genoss. Aber nicht nur die USA kennen eine solche Form der Kandidatenselektion, auch zahlreiche Parteien in Island haben sich für diese Form der Selektion entschieden ${ }^{31}$. Ein weiterer Fall der eher offenen Selektion wäre die katalanische Partei Spanien, welche ihre Kandidatenselektion auch für registrierte Sympathisanten, d. h. Nichtmitglieder, die sich als Parteisympathisanten eintragen lassen, ermöglicht ${ }^{32}$.

Auch bei der Kandidatenselektion über Parteimitglieder finden sich im internationalen Vergleich zahlreiche Variationen ${ }^{33}$. So identifizieren Rahat und Hazan ${ }^{34}$ die Selektion eines Kandidaten durch alle Parteimitglieder und die Selektion eines Kandidaten nur durch Parteimitglieder mit besonderen Eigenschaften (z. B. Höhe der Mitgliedsbeiträge, die Dauer der Parteizugehörigkeit, aktives Engagement innerhalb der Partei etc.). In Belgien finden wir Parteien, in denen Parteimitglieder mit einer bestimmten Parteizugehörigkeitsdauer an dem Prozess der Kandidatenselektion beteiligt sind ${ }^{35}$. In Israel wählen alle Parteimitglieder seit den frühen neunziger Jahren ihre Kandidaten sowie ihren Parteiführer aus ${ }^{36}$. Auch in der Bundesrepublik gab es immer wieder Versuche, die Kandidatenselektion offener zu gestalten. So unternahm die SPD 1994 erstmalig (und bisher auch einmalig) den Versuch, den Parteivorsitzenden durch alle Parteimitglieder direkt wählen zu lassen.

Eine geschlossene Selektion liegt vor, wenn der Kandidat von einem Gremium oder der Parteiführung ernannt wird. Auch hier zeigt die Empirie eine erhebliche Bandbreite an unterschiedlichen Fällen. So finden wir beispielsweise in Großbritannien Parteigremien, die die Möglichkeit haben, Kandidaten zu ernennen bzw. Kandidaturen zu blockieren. Die Parteimitglieder der Liberal Democrats selektieren ihre Kandidaten, die Parteiführung hat allerdings das letzte Wort hierüber. Die Fra-

29 Frank Brettschneider, Spitzenkandidaten und Wablerfolg, Opladen 2002, S. 69 f.

30 Lawrence Leduc, »Democratizing Party Leadership Selection « in: Party Politics, Vol. 7, 3/2001, S. 323-341, hier S. 326.

31 Svanur Kristjansson, »Electoral Politics and Governance: Transformation of the Party System in Iceland 1970-1996 in: Paul Pennings / Jan-Erik Lane (Hg.), Comparing Party System Change, London 1998, S. 167-181.

32 Jonathan Hopkin, »Bringing the Members Back in: Democratizing Candidate Selection in Britain and Spain « in: Party Politics, Vol. 7, 3/2001, S. 343-361.

33 Reuven Y. Hazan, »The 1996 Intra-Party Elections in Israel: Adopting Party Primaries « in: Electoral Studies 16 (1997), S. 95-102; Kristjansson, aaO. (FN 31).

34 Rahat / Hazan, aaO. (FN 23).

35 Lieven de Winter, »Belgium: Democracy or Oligarchy?« in: Michael Marsh / Michael Gallagher (Hg.), Candidate Selection in Comparative Perspective: The Secret Garden of Politics, London 1988, S. 20-46.

36 Hazan, aaO. (FN 33). 
ge nach den Kanzlerkandidaten der beiden Volksparteien in der Bundesrepublik lässt sich auch eher am rechten Ende des Kontinuums festmachen. Zwar werden die Kanzlerkandidaten auf einem Bundesparteitag formal festgesetzt, allerdings wird die Frage, wer kandidieren soll, in informellen Gesprächen in der Parteispitze frühzeitig - allerdings mit einem festen Blick auf die Umfragewerte der in Frage kommenden Kandidaten - geklärt. Erstaunlich ist, dass das Verfahren zur Auswahl der Kanzlerkandidaten noch nicht einmal in den Parteisatzungen von SPD und CDU festgelegt ist. Zudem ist - anders als beispielsweise in Großbritannien - der bzw. die Parteivorsitzende nicht automatisch auch Spitzenkandidat seiner bzw. ihrer Partei bei den Bundestagswahlen ${ }^{37}$.

Aus der bisherigen Diskussion lassen sich also für die Rekrutierung und Selektion der Kandidaten folgende Ausprägungen ableiten:

- Für die Rekrutierungsdimension lässt sich als Extremausprägung auf der einen Seite die offene Rekrutierung festmachen, die sich durch ein breiteres Pool an möglichem Personal auszeichnet. Dem gegenüber steht die geschlossene Rekrutierung, die von den Kandidaten schon eine vergleichsweise längere politische Karriere innerhalb der Organisation verlangt.

- Auf der Dimension der Selektion - also der Frage: wer stellt die Kandidaten letztendlich auf, wer wählt aus dem Pool von Kandidaten aus - lässt sich als Extremausprägung die offene Selektion, d. h. »offene Wahl « für alle Wähler auf der einen Seite aufführen; dem gegenüber steht die zentrale Führungsauslese »von oben«. Die Führungsriege einer Partei einigt sich dabei auf einen Kandidaten und/oder auf eine Kandidatenliste. Wir sprechen hier - analog zu den bisher verwendeten Begrifflichkeiten - von geschlossener Selektion.

\section{Kandidatentypen und Führungseigenschaften}

Nach der Herleitung der zwei Basisdimensionen des Auswahlverfahrens fragen wir im folgenden Schritt nach ihren Eigenschaften. Ausgehend von einem institutionalistischen Ansatz wird angenommen, dass unterschiedliche Rekrutierungs- und Selektionsmechanismen unterschiedliche Kandidateneigenschaften hervorbringen. Die Identifizierung der Führungsqualitäten bzw. idealtypischen Eigenschaften der hier vorgestellten Kandidatentypen ergibt sich zu einem erheblichen Teil aus der Wahrnehmung ihrer Eigenschaften. Hier bedient sich die vorliegende Studie neben den klassischen Ausführungen Max Webers vor allem der Ergebnisse der Wahlforschung. Während in der amerikanischen Wahlforschung zahlreiche Arbeiten zur Beurteilung von Kandidaten vorliegen, ist dieses Forschungsgebiet von der europäischen Politikwissenschaft, respektive der europäischen Wahlforschung, bisher kaum berücksichtigt worden.

Max Webers Arbeiten über die Rolle und Bedeutung eines »Führers « sowie der Differenzierung von Politikern, die »von der Politik« leben, im Gegensatz zu Politi-

37 Brettschneider, aaO. (FN 29), S. 66. 
kern, die »für die Politik leben «, und die in diesen Arbeiten entwickelten »rationalen « und »charismatischen « Führungsqualitäten haben nichts an Aktualität eingebüßt. So führt Weber bei seinen vergleichenden Studien zum Funktionieren von Organisationen die Unverzichtbarkeit der rationalen fachlichen Spezialisierung auf, welche in erster Linie Beamte aufweisen: »Der moderne Beamte ist entsprechend der rationalen Technik des modernen Lebens stetig und unvermeidlich zunehmend fachgeschult und spezialisert. «38 Ein »leitender Geist «, d. h. ein Politiker mit charismatischen Eigenschaften, ist dieser Beamte hingegen nicht. Ein wahrer Führer muss die Macht der demagogischen Rede besitzen, ihn zeichnen »Leidenschaft, Verantwortungsgefühl und Augenmaß aus $^{39}$.

Der Klassiker der amerikanischen Wahlforschung »The American Voter « differenziert zwischen den persönlichen Qualitäten (integer, sympathisch, vertrauensvoll, intaktes Familienleben) und politischen Fähigkeiten (führungsstark, erfahren, unabhängig). Eine inhaltlich ähnliche Unterscheidung wird mit den Bezeichnungen »rollenferne« und »rollennahe « Eigenschaften getroffen ${ }^{40}$. Klein $/ \mathrm{Ohr}^{41}$ sprechen von rollenrelevanten und rollenfernen Kandidatenmerkmalen. Politische, d. h. rollennahe Eigenschaften beinhalten im allgemeinen Managementaufgaben. "Since the voter has only limited information he may vote for a candidate who seems capable of managing the country even if that candidate is not the sclosest< to his specific issue preferences. $\aleph^{42}$ Der Kandidat wird als Problemlöser gesehen ${ }^{43}$. Die persönlichen bzw. rollenfernen Eigenschaften eines Kandidaten dienen den Bürgern häufig als information-shortcut zur Einschätzung und Beurteilung. Jeder Mensch ist täglich aufs Neue gezwungen, sich in Alltagssituationen einen Eindruck von anderen zu bilden und dabei diesen Eindruck auf oft nur minimale Informationen zu gründen. Wenn jeder Bürger im Alltagsleben gewohnt ist, seine Mitmenschen auf Grund ihres Aussehens, ihrer Art zu sprechen und zu argumentieren zu beurteilen, warum sollte dies nicht auch für die Beurteilung von Politikern gelten? Hier führen Klein/Ohr ${ }^{44}$ die Integrität und die Vertrauenswürdigkeit eines Politikers sowie physische Attraktivität als persönliche bzw. rollenferne Merkmale auf. Ob ein Mensch vertrau-

38 Max Weber, Gesammelte Politische Schriften, hg. von Johannes Winckelmann, 5. Aufl. Tübingen 1988, S. 331.

39 Ebd., S. 402.

40 Vgl. u. a. Jürgen Lass, Vorstellungsbilder über Kanzlerkandidaten. Zur Diskussion um die Personalisierung von Politik, Wiesbaden 1995, S. 60; Hans Mathias Kepplinger I Hans-Bernd Brosius / Stefan Dahlem, »Charakter oder Sachkompetenz von Politikern: Woran erinnert sich der Wähler?« in: Hans-Dieter Klingemann / Max Kaase (Hg.), Wablen und Wäbler. Analysen aus Anlass der Bundestagswabl 1990, Opladen 1994, S. 472-505.

41 Markus Klein / Dieter Ohr, »Gerhard oder Helmut? >Unpolitische< Kandidateneigenschaften und ihr Einfluss auf die Wahlentscheidung bei der Bundestagswahl 1998 « in: Politische Vierteliabresschrift 41 (2000), S. 199-224.

42 Samuel Popkin / John W. Gorman / Charles Philips / Jeffrey A. Smith, »Comment: What Have You Done For Me Lately? Toward an Investment Theory of Voting « in: American Political Science Review 70 (1976), S. 779-805, hier S. 794.

43 Lass, aaO. (FN 40).

44 Klein / Ohr, aaO. (FN 41), S. 204. 
enswürdig ist, wird umso bedeutsamer, je unsicherer wir über sein zukünftiges Handeln sind: »We care more about sincerity and character when we are uncertain about what someone will do. «45

Einige Arbeiten führen überzeugend auf, dass diese beiden Dimensionen weiter aufgefächert werden können. So unterscheiden Miller et al. ${ }^{46}$ zwischen den Eigenschaftsdimensionen Kompetenz, Integrität, Verlässlichkeit, Charisma, und primär apolitischen Persönlichkeitsmerkmalen. Kinder et al. ${ }^{47}$ führen ähnliche Dimensionen auf. Vetter/Brettschneider ${ }^{48}$ führen auf der Suche nach dem »Idealmaß « für Kanzlerkandidaten elf Eigenschaften von Kandidaten auf, die die mittlerweile in der Forschungsliteratur dominierenden Dimensionen zur Bewertung von KandidatenMerkmalen abdecken. Die erste Dimension ist die der Issue-Kompetenz mit den Eigenschaften, Konzepte zur Bewältigung der anstehenden Probleme entwickelt zu haben. So muss ein erfolgreicher Kandidat das Image besitzen, die zentralen Probleme lösen zu können. Traut man ihm die Lösung dieser Probleme zu, dann mag außerdem auch einiges dafür sprechen, dem Kandidaten Kompetenz bei der Lösung weiterer Probleme zuzuschreiben, sozusagen als Vertrauensvorschuss: »... numerous problems will emerge during a candidate's term of office that he will have to solve but that neither he nor the voters can anticipate on election day. Competence in unfamiliar areas may be inferred from the perceived competence of the candidate in other areas. $\ll^{49}$ Die zweite Dimension beschreibt die der Manager-Fähigkeiten mit den Eigenschaften Führungsstärke, Entscheidungsfreude und Tatkraft. Persönliche, apolitische Eigenschaften umfassen die dritte Dimension, hier spielen der Geschmack des Kandidaten und seine Ausstrahlung eine zentrale Rolle sowie die Frage, ob er menschlich sympathisch erscheint. Als vierte und letzte Dimension führen Vetter/Brettschneider die der Integrität auf. Hier sind drei Eigenschaften zentral: Ehrlichkeit, Vertrauen und Verantwortungsbewusstsein.

Die in erster Linie von der Verhaltens- und Wahlforschung entwickelten und hier nur in Kürze vorgestellten Kandidateneigenschaften sollen nun mit den sich aus der Basisdimension der Auswahlverfahren ergebenden gekoppelt werden. Welche Kandidatentypen ergeben sich also aus den Dimensionen und welchem Kandidatentyp lassen sich welche Führungsmerkmale bzw. Eigenschaften zuordnen?

Die Zusammenführung der beiden Dimensionen Selektion und Rekrutierung ergibt eine Kandidatentypologie (vgl. Tabelle 1).

45 Samuel Popkin, The Reasoning Voter. Communication and Persuasion in Presidential Campaigns, Chicago 1991, S. 65.

46 Arthur H. Miller / Martin P. Wattenberg / Oksana Malanchuk, »Schematic Assessments of Presidential Candidates « in: American Political Science Review 80 (1986), S. 521-540.

47 Donald R. Kinder, »Presidential Character Revisited « in: Richard Lau / David O. Sears (Hg.), Political Cognition, Hillsdale 1986, S. 233-255.

48 Angelika Vetter / Frank Brettschneider, »Idealmaße< für Kanzlerkandidaten « in: ZUMA-Nachrichten 43, 22/1998, S. 90-115.

49 Popkin / Gorman / Philips / Smith., aaO. (FN 42), S. 794. 
Tabelle 1: Kandidatentypen

\begin{tabular}{|c|c|c|c|}
\hline \multirow{4}{*}{ 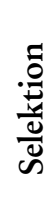 } & & \multicolumn{2}{|c|}{ Rekrutierung } \\
\hline & & Offen & Geschlossen \\
\hline & offen & $\begin{array}{l}\text { Charismatischer } \\
\text { Politiker }\end{array}$ & Parteimanager \\
\hline & geschlossen & Seiteneinsteiger & Funktionär \\
\hline
\end{tabular}

\section{Funktionär}

Der Funktionär ist geschlossen rekrutiert, d. h. er durchläuft innerhalb der Partei zahlreiche Posten und Funktionen; der Weg durch die bundesrepublikanischen Parteien wird hier treffend als die »Ochsentour « bezeichnet. Der Funktionär beginnt seine politische Laufbahn sehr frühzeitig in besoldeten politischen Berufstätigkeiten. Er wird zudem geschlossen selektiert. Generell lässt sich dieser Auswahlmechanismus als Beförderung (Promotion) beschreiben. Der Funktionär verfügt über Sachkenntnis, Expertenwissen, Detailinformation. Er ist ein politischer Professional, d. h. ein Politiker, der von der Politik lebt und die Weberschen rationalen Führungseigenschaften par excellence verkörpert. Da er sich keiner Wahl stellen muss, sind apolitische, rollenferne Eigenschaften bei diesem Kandidatentypus nicht notwendigerweise zu erwarten.

\section{Parteimanager}

Zwar zeichnet auch den Parteimanager eine längere Parteikarriere aus, im Unterschied zum Funktionär kommt er allerdings durch eine offene Selektion in wichtige politische Ämter. Während der Funktionär keine externe Legitimation hat und auch keine Verbindung aus der Partei heraus, ist die parteiexterne Unterstützung für den Parteimanager notwendige Bedingung. Wir nennen diesen Auswahlmechanismus Nominierung. Die offene Selektion fordert ohne Zweifel charismatische Eigenschaften, der Kandidat muss auch außerhalb seiner Partei für Unterstützung werben. Hier spielen, um in der Terminologie der Wahl- und Verhaltensforscher zu sprechen, auch apolitische bzw. persönliche Qualitäten wie Ansehen, Integrität eine zentrale Rolle. Da Politik zunehmend komplexer und somit auch schwerer vermittelbar ist, suchen Bürger bei ihrer Wahlentscheidung nach einem information-shortcut. Eine Möglichkeit zur Vereinfachung komplexer Sachverhalte besteht darin, die Auswahl aus mehreren Optionen auf Kriterien zu stützen, die aus dem Alltagsleben vertraut sind, und hierzu gehören persönliche Eigenschaften. Zugespitzt formuliert kann gesagt werden, dass hier sachpolitische Erwägungen in den Hintergrund treten (obwohl der Kandidat aufgrund der geschlossenen Rekrutierung über Fachwissen verfügt!). Aufgrund seiner geschlossenen Rekrutierung kann aber, in Anlehnung an die Arbeiten von Korte ${ }^{50}$, auch ein korporatistischer Führungsstil erwartet 
werden, d. h. das Verhandeln am »Runden Tisch« wird zur Dauereinrichtung. Der Parteimanager sucht den Konsens aller Beteiligten durch Integration.

\section{Seiteneinsteiger}

Ein Seiteneinsteiger wird offen rekrutiert und von der Parteispitze ernannt. Diesen Auswahlmechanismus nennen wir appointment. Der Seiteneinsteiger zeichnet sich durch sein Fachwissen auf einem ganz bestimmten Gebiet aus. Herzog spricht hier von »Cross-over-Karrieren«, wobei es sich nicht um eine Karriere im eigentlichen Sinne handelt, sondern um einen Positionssprung aus einer führenden Funktion im nicht-politischen Beruf in eine führende Funktion der Politik. Auf den ersten Blick hat diese Art von Führungsrekrutierung durchaus positive Folgen: eine größere Flexibilität im Personalauswahlprozess, die mögliche Gewinnung prominenter, auch eigenwilliger Persönlichkeiten, die sich den Bedingungen einer partei-politischen Ochsentour nicht unterwerfen mussten. Doch stehen dieser Rekrutierungsweise oftmals verschiedene parteiorganisatorische und wahlrechtliche Bedingungen entgegen. Was sind nun aber die zentralen idealtypischen Eigenschaften eines Seiteneinsteigers? Ein Seiteneinsteiger zeichnet sich durch seine Expertise in einem bestimmten Bereich aus, er verfügt auf seinem Fachgebiet über einen großen Erfahrungswert, über die notwendigen Kontakte sowie über großes Ansehen und Anerkennung. Zudem zeichnet ihn im politischen Geschäft eine gewisse Unabhängigkeit aus; partei-interne Streitigkeiten und Befindlichkeiten sind ihm fern, ferner hat er aufgrund seiner offenen Rekrutierung nach Ablauf seiner Amtszeit oder bei Unstimmigkeiten natürlich auch schon während seiner Amtszeit die Möglichkeit, in seine frühere nicht-politische Tätigkeit zurückzukehren. Da der Seiteneinsteiger keiner externen Legitimation bedarf, sind apolitische, rollenferne Eigenschaften keine Bedingung; allerdings kann die Unterstützung der öffentlichen Meinung (die über rollenferne Eigenschaften eingeworben werden kann) eventuell auftretende partei-interne Querelen und Unstimmigkeiten ausbügeln.

\section{Charismatische Politiker}

Der charismatische Politiker, der Demagoge, ist offen rekrutiert, d. h. es bedarf keiner (langen) Laufbahn innerhalb der Parteiorganisation, um in das Pool der potenziellen Kandidaten zu gelangen. Ein charismatischer Politiker kann vor seiner politischen Laufbahn durchaus anderen Tätigkeiten nachgegangen sein und dann, ähnlich dem Seiteneinsteiger, eine Cross-over-Karriere in die Politik anstreben. Max Weber sieht dies schon fast als Voraussetzung für den charismatischen Politiker: »Was in aller Welt soll dagegen eine Partei für eine Anziehungskraft auf Männer mit

50 Karl-Rudolf Korte, »Veränderte Entscheidungskultur: Politikstile der Deutschen Bundeskanzler « in: Gerhard Hirscher / Karl-Rudolf Korte (Hg.), Darstellungspolitik oder Entscheidungspolitik? Über den Wandel von Politikstilen in Westlichen Demokratien, München 2001, S. 14-29. 
Führungsqualitäten ausüben? Welche Gelegenheit bietet sie ihnen denn, solche zu entfalten? « Im Gegensatz zum Seiteneinsteiger wird der charismatische Politiker jedoch offen selektiert, d. h. er muss sich einer Wahl stellen. Diesen Auswahlmechanismus nennen wir Akklamation. Welches sind die zentralen Eigenschaften dieses Kandidatentypus? Um mit Weber zu sprechen, kann man diesem Politikertyp einen unabdingbaren Willen zur Macht, der durch langjährige Parteiarbeit noch nicht abgeschliffen ist, und Verantwortungsbewusstsein zusprechen. Er ist nicht in erster Linie ein Spezialist auf einem bestimmten Gebiet, sondern kann vielmehr als Allroundtalent bezeichnet werden. Hinzu kommen - wie es bei Weber heißt - »massendemagogische« Eigenschaften. Wir würden heute eher sagen, dass dieser Politikertyp, um die Unterstützung der Wähler zu erhalten, medien- und publikumswirksam auftreten muss. Er muss aufgrund der Direktwahl breite Massen für sich mobilisieren können, er muss mit den Wählern in Kontakt stehen, auf ihre Interessen und Forderungen eingehen. Ziehen wir die oben aufgeführten Studien zu Rate, so sind es vor allem die apolitischen Eigenschaften, die diesen Idealtyp auszeichnen. Sein Charisma und seine Ausstrahlung sind von zentraler Bedeutung. Wie zahlreiche empirische Studien aus der Wahlforschung belegen, orientieren sich die Wähler bei ihrer Wahlentscheidung durchaus an diesen Eigenschaften. So fassen Klein/Ohr die Ergebnisse ihrer empirischen Untersuchung zum Einfluss auf das Wahlverhalten bei der Bundestagswahl 1998 folgendermaßen zusammen: »Besonders die persönliche Vertrauenswürdigkeit, aber auch das Privatleben und die physische Attraktivität der Kandidaten tragen signifikant zur Erklärung des berichteten Wahlverhaltens bei. «1 Im Gegensatz zum Parteimanager, der zwar auch offen selektiert, aber geschlossen rekrutiert wird, kann bei dem charismatischen Politiker nicht von einer umfangreichen Kenntnis des politischen Betriebes ausgegangen werden. Im Extremfall ist er ein Neuling im politischen Geschäft ${ }^{52}$.

\section{Kandidatentypen und die Qualität politischer Fübrung}

Die Studie hat bisher unterschiedliche Rekrutierungs- und Selektionsmechanismen identifiziert, aus denen sich unterschiedliche idealtypische Kandidaten und Kandidateneigenschaften ableiten lassen. Im folgenden Schritt sollen nun diese Eigenschaften kritisch diskutiert werden. Aus diesem Grunde müssen Kriterien definiert werden, die zur Beurteilung dieser Merkmale herangezogen werden können. Und hier schlagen wir wieder den Bogen zu der eingangs aufgeführten Definition von politischen Eliten: Die Antwort auf die Frage nach der Qualität politischer Führung ist aus den Funktionen abzuleiten, die politische Eliten in repräsentativen Demo-

51 Klein / Ohr, aaO. (FN 41), S. 219.

52 Zur empirischen Erhebung könnten folgende Informationen (aus Datenhandbüchern und in selteneren Fällen über Interviews) herangezogen werden: seit wann Mitglied in der Partei, Einstiegsalter in die Politik (d. h. Politik als Beruf), politiknahe oder politikferne Ausbildung (d. h. Fachwissen oder Allroundwissen), Verweildauer in anderen Berufen, Anzahl der Posten innerhalb einer Partei. 
kratien zu erfüllen haben. Die politikwissenschaftliche Literatur bietet hier die unterschiedlichsten Funktionskataloge an, aber es sind im Hinblick auf die Rolle und Funktion von Eliten in repräsentativen Demokratien zwei ganz zentrale Funktionen zu nennen:

- aus einer eher funktionalistischen Perspektive her betrachtet die der Effektivität und

- aus einer demokratietheoretischen Perspektive her gesehen die der Responsivität.

Wenden wir uns zuerst der Effektivität zu. Damit ist die Fähigkeit der politischen Akteure gemeint, Probleme zu erkennen, zu lösen, Entscheidungen zu treffen und diese zugleich zu verantworten. Hierzu sind in komplexen, hochgradig differenzierten Gesellschaften Polit-Profis, Berufspolitiker mit Detailwissen und Sachkenntnis, d. h. mit rationalen Führungsqualitäten, gefragt. Waschkuhn brachte dies vor einiger Zeit auf folgende Formel: »Generell ist festzustellen, dass politische Eliten im funktionalen Sinn für hochdifferenzierte Gesellschaften unverzichtbar sind; sie bedürfen jedoch der Legitimation, und ihre Verselbständigung oder 'Selbstperpetuierung< ist zu verhindern. ${ }^{53}$ Auch Otto Stammer begründete aus dieser Perspektive die Notwendigkeit von Eliten in modernen Demokratien. »Angesichts der vorerst unüberwindlichen großen bürokratisch funktionierenden Apparaturen ist das Volk in seiner Gesamtheit überhaupt nicht in der Lage, den politischen Willen in der bei der zunehmenden Komplizierung der sozialen und politischen Entscheidungen erforderlichen Gründlichkeit und Präzision ohne die Hilfe sozial und politisch aktiver Minderheiten zu bilden. ${ }^{54}$

Aus demokratietheoretischer Perspektive ist - wie bereits erwähnt - eine weitere Funktion die der Responsivität. Responsivität (responsiveness) ist ein Begriff, der in der amerikanischen Politikwissenschaft in den späten fünfziger Jahren geprägt wurde: Hierunter wird das den Einstellungen der Bürger entsprechende Handeln der Abgeordneten und der Regierung verstanden. Voraussetzung dafür ist, dass Akteure die Interessen und Forderungen der Bürger kennen, sie aufnehmen und in ihr inhaltliches Angebot verarbeiten. Dies setzt einen Dialog mit den Bürgern voraus, d. h. diese Perspektive betont eher partizipatorische Elemente des demokratischen Prozesses $^{55}$. Responsivität fordert von den politischen Akteuren auch charismatische Führungsqualitäten, d. h. sympathische Erscheinung und Eloquenz sind hier gefragt.

Bei der Frage nach den Führungsmerkmalen der einzelnen Kandidatentypen muss nun immer gefragt werden, inwieweit sie einen Beitrag zur Erfüllung der aufgeführten Funktionen leisten. Die bisherige Diskussion lässt erkennen, dass die ge-

53 Arno Waschkuhn, "Sind Eliten (Un)Demokratisch, oder: Wie gut >bemannt< sollten Institutionen sein? « in: Wolfgang Luthardt / Arno Waschkuhn (Hg.), Politik und Repräsentation. Beiträge zur Theorie und zum Wandel Politischer und Sozialer Institutionen, Marburg 1988, S. 14-32, hier S. 29.

54 Stammer, aaO. (FN 3), S. 75.

55 Vgl. auch Amitai Etzioni, The Active Society. A Theory of Societal and Political Processes, London 1968. 
schlossene Rekrutierung den politischen Akteuren die notwendige Expertise, das Fachwissen, das Agieren und Handeln im komplexen Geflecht der politischen Organisationen und Institutionen vermittelt. Die Effektivität eines politischen Systems ist demnach am ehesten durch politische Akteure gewährleistet, die rationale, d. h. politische Führungsqualitäten besitzen. Hier sind der Seiteneinsteiger sowie der Funktionär zu nennen. Responsivität setzt eine Verbindung, ein linkage zwischen den politischen Akteuren und den Wählern voraus. Eine offene Selektion gewährleistet über die Wahl eine solche Responsivität. Ein Wähler selektiert den Kandidaten, von dem er annimmt, dass dieser seine Belange und Forderungen am ehesten repräsentiert. Möchte der Kandidat eine möglichst große Zahl an Stimmen auf sich vereinen, muss er sich in hohem Maße responsiv verhalten. Unserer Kandidatentypologie entsprechend sind dies hier der Parteimanager und der charismatische Politiker, die beide aufgrund der externen Legitimation auch über charismatische, d. h. apolitische Qualitäten verfügen müssen.

\section{Repräsentative Demokratien und politische Elite}

Die Studie hat bisher einen Zusammenhang von Auswahlmechanismus und Kandidateneigenschaften hergestellt. Die Arbeit identifiziert

- den charismatischen Politiker (offen rekrutiert und offen selektiert - Akklamation),

- den Parteimanager (geschlossen rekrutiert und offen selektiert - Nomination),

- den Seiteneinsteiger (offen rekrutiert und geschlossen selektiert - Appointment)

- sowie den Funktionär (geschlossen rekrutiert und geschlossen selektiert Promotion).

Die Bewertung ihrer Eigenschaften - d. h. die Frage nach ihrer Qualität - erfolgte aus einer Ableitung der Funktionen politischer Eliten in repräsentativen Demokratien und hat das Demokratien innewohnende Spannungsverhältnis zwischen Effektivität und Responsivität erneut verdeutlicht.

Die Frage, welchem Nominierungsverfahren (und somit welchen Führungsqualitäten) der Vorzug zu geben sei, drängt sich abschließend auf. Der immer wieder in der aktuellen politischen Diskussion lautwerdende Ruf nach einer Reform der Nominierungsverfahren ist in den meisten Fällen mit einer Forderung nach mehr Partizipation der Bürger verbunden, d. h. bricht die Lanze für den Responsivitätsaspekt eines politischen Systems. In den siebziger Jahren wurden diese Forderungen unter dem Stichwort der innerparteilichen Demokratie diskutiert, in den neunziger Jahren standen diese Forderungen im Zeichen der Personalisierung von Politik. Dabei gilt es generell zu beachten, dass für verschiedene politische Ämter unterschiedliche Qualitäten erforderlich sind. Darüber hinaus kann es im Verlauf einer politischen Karriere durchaus von Nöten sein, verschiedene Ämter und damit verschiedene 
Auswahlverfahren zu durchlaufen, die dann die unterschiedlichen Qualitäten bei den Eliten fordern.

Aus funktionalistischer Perspektive steht in modernen, hochgradig differenzierten Gesellschaften der Bedarf an Profis, an gelernten Politikern mit dem notwendigen Rüstzeug für das komplexe politische Alltagsgeschäft im Mittelpunkt. Ein hoher Grad an Professionalisierung gewährleistet am ehesten die Problemlösungskompetenz der politischen Elite. Die Notwendigkeit von rationalen, politischen Führungsqualitäten ist hier Trumpf. Politische Parteien sind - sicherlich mit einigen wenigen Ausnahmen und Einschränkungen - der Ort, sich diese Qualitäten anzueignen. Trotz aller Kritik an den damit verbundenen Parteiverkrustungserscheinungen bleibt dies ein zentrales Argument. Effektiviät eines politischen Systems kann nur durch eine eher geschlossene Rekrutierung gewährleistet sein.

Es bleibt letztendlich eine empirisch zu überprüfende Frage, welche Posten und welche Ämter wie besetzt werden und welchem Verfahren sich die Kandidaten dabei unterziehen müssen.

\section{Zusammenfassung}

Bringen unterschiedliche Nominierungsverfahren für politische Ämter unterschiedliche Führungsqualitäten hervor und wenn ja, welche Bedeutung hat dies für die repräsentative Demokratie? So lautet die zentrale Forschungsfrage der vorliegenden Analyse. In einem ersten Schritt werden die Begriffe der Elitenrekrutierung und -selektion begrifflich klar definiert und voneinander abgegrenzt. In einem zweiten Teil diskutiert der Beitrag theoretisch unterschiedliche Mechanismen zur Rekrutierung und Selektion politischer Kandidaten und entwickelt hieraus eine Kandidatentypologie. Mit Zuhilfenahme der Ergebnisse der Wahlforschung werden die sich aus der unterschiedlichen Rekrutierung und Selektion ergebenden Führungseigenschaften der Kandidaten in einem dritten Teil präsentiert. Der Beitrag schließt mit einer Diskussion über unterschiedliche Führungsqualitäten in repräsentativen Demokratien.

\section{Summary}

Do different methods of elite recruitment bring about different leadership qualities and if so, what are the consequences for representative democracy? This is the central research question of the study presented. We start our study with a clear definition of elite recruitment and elite selection. In a second part the analysis discusses different mechanisms of candidate recruitment and selection resulting in a candidate typology. By taking results of electoral research into account, we propose various leadership qualities (Eigenschaften) resulting from different recruitment and selection processes. Finally, we discuss the role of different leadership qualities in representative democracies. 